\title{
ETNOZOOLOGÍA, USOS Y ABUSOS DE LOS CUESTIONARIOS
}

\section{Celeste MEDRANO ${ }^{1}$}

\section{Resumen}

La etnozoología es una ciencia interdisciplinaria cuyo cuerpo metodológico se construye gracias al aporte de los métodos de las ciencias naturales y humanas. En este trabajo se discuten y amplían los alcances prácticos que otorgamos a los cuestionarios. Se aportan cuestionarios para trabajo en campo elaborados en base a la experiencia metodológica adquirida. Se discute sobre el uso de estímulos visuales y la necesidad de colectas biológicas. Finalmente se analizan aspectos relacionados al valor relativo de los instrumentos de recolección de datos.

Palabras claves: etnozoología, metodología, cuestionarios, tobas del Gran Chaco.

\begin{abstract}
:
Ethnozoology is an interdisciplinary science whose methodological body is constructed due to natural and human sciences methods. In this paper we discuss and extend the practical scope we give to the questionnaires. These are provided for field work and are based on the methodological

${ }^{1}$ Licenciada en Biodiversidad. Becaria CONICET. Instituto de Ciencias Antropológicas, Universidad de Buenos Aires.. celestazo@hotmail.com
\end{abstract}

Fecha de recepción del artículo:Marzo 2012

Fecha de evaluación:Aril 2012 
experience gained. We discuss the use of visual stimuli and the need for biological collections. Finally, issues related to the relative value of the data collection instruments are analized.

Keywords: ethnozoology, methodology, questionnaires, tobas of the Gran Chaco.

\section{Résumé}

L'Ethnozoologie est une science interdisciplinaire dont le corps méthodologique est construit à partir des méthodes des sciences naturelles et humaines. Dans ce texte, nous discutons la portée pratique que nous donnons à ces questionnaires. Ceux-ci sont élaborés pour le travail de terrain préparé à partir de l'expérience méthodologique acquise. On discute l'utilisation de stimuli visuels et le besoin des collections biologiques. Enfin, on discute certaines questions liées à la valeur relative des instruments de collecte de données.

Mots-clés: ethnozoologie, méthodologie, questionnaires, tobas du Gran Chaco.

\section{INTRODUCCIÓN}

Cada sociedad se relaciona con su "entorno natural" de una manera singular y desarrolla formas particulares de conocer, manejar, utilizar, visualizar y percibir la naturaleza que difieren del modo en que la ciencia occidental lo hace.

Esta diversidad de saberes suelen ser estudiados tanto por las disciplinas tradicionales como por las etnociencias, en particular, la etnobiología. El prefijo etno- se refiere al sistema de conocimientos y "percepciones propias" de determinada cultura. De este modo, la etnobiología se define como el análisis de lo que una comunidad conoce, denomina, utiliza, cree, clasifica y piensa acerca del entorno biológico que la rodea (Posey, 1987; Toledo, 1990 y 1991; Albuquerque, 1999). Esta disciplina funciona como nexo entre los conocimientos vernáculos y la ciencia occidental, ya que se propone registrar los sistemas simbólicos y las prácticas que poseen las diversas sociedades en relación con el entorno natural. Así también, tiene por objetivo analizar los roles que dicho entorno desempeña en el contexto sociocultural de las sociedades en cuestión.

La etnobiología nace como ciencia a finales del siglo XIX. En el proceso de formación y consolidación de la disciplina pueden distinguirse tres fases denominadas: preclásica, clásica y posclásica (Clément, 1998). Los primeros estudios se centran en el modo en que las sociedades catalogadas como "primitivas" aprovechan plantas y animales. Para ello se elaboran listados descriptivos con nombres vernáculos y sus equivalentes en la taxonomía académica (Brown, 1868; Palmer, 1878; Mason, 1989; Henderson y Harrington, 1914; Robins et al., 1916 y Castetter, 1935). En la fase clásica, un nuevo movimiento antropológico de tendencia cognitivista junto con 
la sociolinguística renueva el carácter de las investigaciones y las nuevas preocupaciones giran en torno a cómo los sujetos nombran y ordenan los elementos de su ambiente en sistemas clasificatorios. Sin embargo, estos estudios reciben fuertes críticas por el hecho de separar los saberes tradicionales de sus significados prácticos (Clément, 1998 y Hunn, 2007). A partir de 1980, en función de estas críticas, comienza la fase posclásica en la que los estudios contemplan la perspectiva de los sujetos de la investigación, tanto de su realidad sociocultural como de su relación con el ambiente. Dicho período se caracteriza por una mayor cooperación entre científicos y poblaciones indígenas, campesinas, pescadores locales, etc. (Towsend, 1996 y Ulloa et al., 1996). Los actuales estudios etnobiológicos integran el conocimiento con la práctica, sin desestimar el beneficio que esto supone para los mismos conocedores, incluso como estrategia de revalorización y sustento de su identidad sociocultural y territorial, entendiendo el lugar habitado como un espacio físico y simbólico (Santos Fita et al., 2009).

Esta breve historia de la etnobiología soslaya el gran esfuerzo que la disciplina emprendió para construir su cuerpo teórico y metodológico particular. Si el período preclásico estuvo dominado por zoólogos y botánicos, el segundo se caracterizó por la producción de antropólogos quienes, criticando los primeros intentos, realizaron el mayor aporte a la producción académica de esa fase. Actualmente, existe un acuerdo generalizado en considerar a la etnobiología como una ciencia interdisciplinaria cuyo cuerpo metodológico se construye gracias al aporte de los métodos tradicionales de las ciencias naturales tanto como de los métodos propios de las ciencias humanas. Sin embargo, el desafió actual consiste en construir un cuerpo teórico-metodológico propio enfrentándose a la urgencia que supone trabajar en comunidades con conflictos sociales, económicos y territoriales para quienes los datos registrados por los investigadores se vuelven centrales para sus demandas. El fin es alcanzar una discusión que nos permita contribuir con el avance y el fortalecimiento de esta "ciencia de los saberes".

Por otro lado, siguiendo las subdivisiones clásicas en ciencias naturales, la disciplina comprende áreas como la etnobotánica -focalizada en el entorno vegetal- y la etnozoología -concentrada en las prácticas y representaciones inherentes al mundo animal-, entre otras tantas, que fueron surgiendo conforme los estudios etnobiológicos fueron avanzando. Así, podemos nombrar, por ejemplo, a la etnomicología, la entnoentomología, la etnoherpetología hasta agotar las extensas denominaciones que se emplean en la academia. El presente trabajo se circunscribe al ámbito de la etnozoología.

Costa Neto et al. definen a la etnozoología como "la ciencia y rama de la etnobiología que estudia el conocimiento zoológico tradicional ${ }^{2}$ (CZT) de aquellos grupos humanos -indígenas, campesinos, pescadores artesanales, entre otros- comúnmente llamados tradicionales (2009: 15)”. Dichos

\footnotetext{
${ }^{2}$ El uso de itálica es del original.
} 
autores expresan, siguiendo a Diegues (2000) que para muchas de estas comunidades tradicionales (sobre todo las indígenas) existe una "interconexión orgánica" entre el mundo natural, el sobrenatural y la organización social. En base a esto, para alcanzar conocimientos sobre la etnozoología de determinado grupo, se necesita comprender el sistema simbólico que organiza la fauna. Resulta clave entender la concepción nativa de lo que es un "animal", definir las fronteras -claras o difusas- que los distinguen de los seres humanos y otros seres no-humanos que muchas veces interactúan con los humanos y animales en las distintas sociedades.

En base a lo expuesto hemos organizado el presente trabajo cuyo objetivo es discutir y ampliar los alcances prácticos que otorgamos a los cuestionarios calificados como uno de los métodos clásicos en etnobiología. Como se aclaró previamente, al tratarse de una ciencia interdisciplinaria, se suelen integrar métodos y técnicas de las ciencias biológicas (ecología, sistemática, taxonomía), sociales (antropología, geografía, historia, sociología psicología) y lingüísticas. Sin embargo, al realizar trabajo de campo, la metodología tradicional consiste en hacer amplias descripciones etnográficas ${ }^{3}$ registrando información que supera el objetivo de la investigación en sí. Dicha información se obtiene generalmente a través de técnicas como la observación participante, el registro de las historias orales, la elaboración de mapas y representaciones gráficas $\mathrm{y}$, fundamentalmente, los cuestionarios.

El cuestionario es considerado una técnica cerrada ya que es construido por el investigador antes de comenzar el trabajo de campo. Según Santos Rodríguez "aunque el cuestionario no sea necesariamente un material compuesto solamente con datos y preparado "en la oficina", acaba restringiendo sustancialmente el trabajo, creando determinadas reglas que van a dirigir las entrevistas, pudiendo ser una barrera más entre entrevistador y entrevistado" (2009: 264). No obstante, el mismo autor aclara que una oportuna flexibilidad en el empleo de las guías puede resolver este problema. Nosotros entendemos que una permanente vigilancia de esta herramienta de recolección de datos, la capacidad de poder reestructurarla inagotablemente a la luz de lo que se obtiene en el campo y la combinación con otras técnicas más elásticas permiten superar la rigidez de algo que tiene la desventaja de ser pergeñado a priori. Por otro lado, es necesario que exista el espacio de opinión tanto del entrevistador como de los entrevistados sobre el cuestionario ya que, en mutua comprensión, ambos "crecen juntos" (labrando el mítico rapport).

$\mathrm{Al}$ respecto, la antropóloga Roxana Guber señala que la mayor limitación de los cuestionarios se presenta cuando "los supuestos del investigador se proyectan en el discurso del otro, cerrando el

\footnotetext{
${ }^{3}$ Hoy en día existe un debate en el campo etnobiológico acerca de los métodos y técnicas que realmente validan el conocimiento y que van de los más cualitativos a los más cuantitativos, "sin embargo, por lo general aún se considera a la etnografía una óptima manera de trabajar la investigación "de campo" o "en campo" (Santos Rodríguez, 2009: 259)".
} 
acceso al universo de sentidos que componen la perspectiva del actor" (2009: 204). Como ejercicio para resolver esta dificultad, la autora propone acompañar al informante por los caminos de su lógica y aclara que "al comenzar la investigación es inevitable cierta dosis mínima de sociocentrismo cuya superación no tiene que ver con adoptar la alternativa empirista, sino con relativizar la propia mentalidad, contrastarla con el campo y desplazarse hacia un reconocimiento de la perspectiva del actor como independiente de la del investigador" (ibid.: 215).

La práctica propuesta por Guber requiere, como vemos, tiempo y, a menudo, en el trabajo de campo se suceden frustraciones y éxitos -en la comprensión del universo del otro- que participan en la construcción de lo que luego serán los "datos". Ante este escenario, y bajo conocimiento de todas sus salvedades, los cuestionarios ordenan, son los aliados cuando la intuición nos abandona y nos permiten vigilar aquellos vacíos que encontramos en el entendimiento de esos "otros" sistemas prácticos y simbólicos. Por todo esto, reivindicamos el uso de los mismos y nos explayaremos sobre la función que la disciplina etnozoológica les ha dado.

\section{CUESTIONARIOS POSIBLES}

Inspirados en los cuestionarios aportados por Thomas (1992) y Venot (1992), a continuación se presentarán cuestionarios de elaboración propia con la finalidad de obtener datos etnozoológicos. Los mismos fueron construidos en base a investigaciones de campo realizadas en comunidades qom (toba) $)^{4}$ en la provincia de Formosa (Argentina), cuyo objetivo fue describir la etnozoología qom. Puntualmente se utilizará la experiencia obtenida en distintos trabajos de campo realizados entre 2008 y 2011 en las colonias "El Desaguadero" y "El Naranjito", los barrios tobas "Nlaxayec" (La Paz) y "12 de octubre" (ubicados dentro del ejido urbano de la ciudad de El Colorado) así como en el barrio periurbano "Namqom" situado a $11 \mathrm{~km}$ de la ciudad de Formosa. A esto se suma la orientación de Mauricio Maidana, quien vive en el barrio toba

\footnotetext{
${ }^{4}$ Los qom pertenecen a la familia lingüística guaycurú. Los guaycurúes eran etnias cazadoras-recolectoras y pescadoras, entre los que estaban abipones, tobas, mocovíes, payaguáes y mbayás (en la actualidad se agregan los grupos pilagás y caduveos). Según Susnik "el término "Guaycurú" es un simple apelativo guaraní para el grupo étnico que vivía en los comienzos de la conquista hispana en la región chaqueña, desde el Río Pilcomayo hasta el Río Verde; en la época colonial se aplicaba este término a diferentes tribus de carácter belicoso y ecuestre; en la moderna etnología el mismo término se introdujo para designar tribus de estrecha filiación lingüística" (1972: 12). En la actualidad, la familia lingüística guaycurú es una de las más extensas del Gran Chaco.
} 
"Daviaxaiqui" (Derqui, provincia de Buenos Aires) y se desempeña actualmente como profesor de qom l'aqtaqa (lengua toba) en distintos ámbitos académicos.

\section{Primeras exploraciones}

La propuesta que se desplegará contiene tres cuestionarios que, según la experiencia de campo, conviene explorar en orden sucesivo para obtener informaciones etnozoológicas. El esquema planteado, pretende ser solo eso; un desarrollo del mejor de los escenarios posibles para abordar una investigación etnozoológica. Sin embargo, llegamos "al campo" de múltiples maneras y nos enfrentamos con hablantes de lenguas -que van de las totalmente conocidas a las totalmente desconocidas- con quienes deberíamos poder compartir el desarrollo de nuestra investigación. Esto nos conduce a evaluar la posibilidad de usar o descartar los cuestionarios, modificarlos y discriminar su uso de acuerdo al caso, la competencia y gusto del hablante.

El primer cuestionario que se describirá aborda la terminología utilizada por los hablantes ${ }^{5}$ para referirse a los aspectos anatómicos, fisiológicos y psicológicos de los animales ${ }^{6}$. Su objetivo es alcanzar un primer marco de conocimiento. Conviene desarrollar esta primera práctica con un hablante que esté familiarizado con las entrevistas y pensar que los datos que se obtengan son claves para precisar futuros diálogos con respecto al mundo animal. No obstante, como venimos insistiendo, muchas veces no hallamos aquella persona con la que extendernos en un cuestionario como el que se presenta a continuación y el problema puede ser salvado tomando como ejemplo a aquellos animales domésticos que se encuentren cercanos.

\section{Cuestionario 1:}

Para el desarrollo de este cuestionario elegimos distintos dibujos de animales que se presentarán sin leyenda a los hablantes. Como modelo aportamos algunos nombres que hemos obtenido con hablantes de qom l'aqtaqa (lengua toba). Estos datos resultan fundamentales en una investigación etnozoológica porque nos permiten manejar la misma terminología y precisar datos. Por otro lado, nos ayudan a definir qué términos se utilizan con exclusividad para animales comparando con aquéllos que se emplean para humanos, plantas, etc.

\footnotetext{
${ }^{5}$ Con fines prácticos, se utilizarán indistintamente los términos hablante e informante para referirnos a aquellas personas con las que trabajamos en campo a pesar de reconocer que existe todo un debate en torno esta denominación.

${ }^{6}$ En el presente trabajo se expondrán cuestionarios enfocados en obtener información sobre mamíferos con el fin práctico de no extendernos demasiado en las recomendaciones. Sin embargo, las propuestas pueden adaptarse al resto de los grupos animales.
} 


\section{1. Atributos físicos}

\section{1. 1. Anatomía externa}

El número de estos dibujos indica varias partes del cuerpo así como expresiones, posturas y posiciones de los animales. Los términos en castellano pueden no tener su equivalente en la lengua nativa y los hablantes pueden incluir términos específicos que no existan en castellano. Por esta razón, el uso de dibujos es preferible a la utilización de cuestionarios no ilustrados. Es importante aclarar que los términos esbozados aquí no constituyen una lista cerrada; la misma se ampliará según las necesidades de la investigación y el avance de la misma.

- Términos usado para macho y hembra, cría.

- Cabeza: cuernos o astas (FIGURA 1), oído, frente, puente de la nariz, punta de la nariz, ventana de la nariz, mentón, bigotes, boca, encías, labios, lengua, dientes (diferentes tipos) (FIGURA 2), mejilla, mandíbula inferior, ojos, párpados, pestañas, cuello.

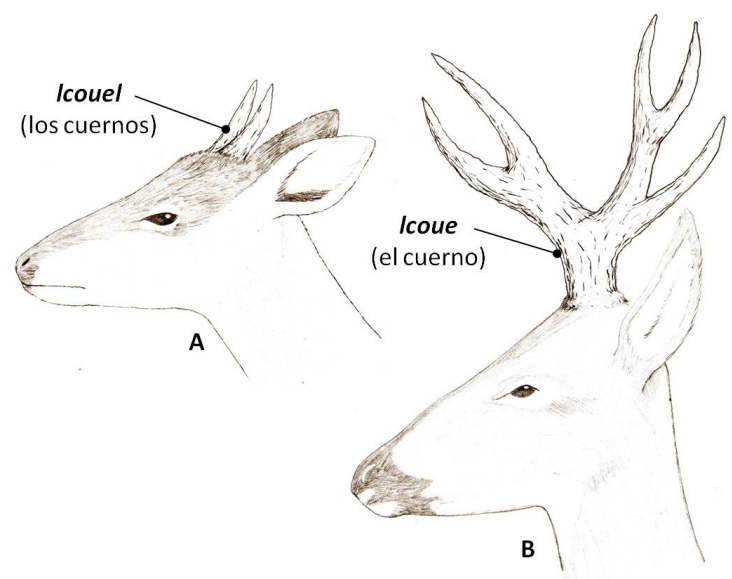

FIGURA 1: Distintos tipos de cuernos. A: Mazama sp.; B: Blatoceros dichotomus. (Dibujo: Ruy Gatti) 


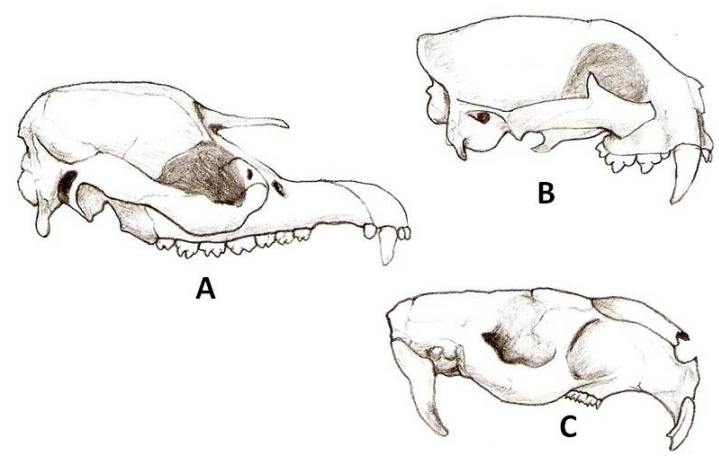

FIGURA 2: Distintos tipos de dientes en cráneos de mamíferos. A: Tapirus terrestris; B: Puma concolor; C: Hydrochaeris hydrochaeris. (Dibujo: Ruy Gatti)

- Cuerpo (FIGURA 3): pelos, cuero, caparazón (Ejemplo Dasypodidos: mulitas, quirquinchos, armadillos), alas (Ejemplo Quirópteros: murciélagos) pecho, hombro, vientre, flanco, cola, lomo, cuello, cresta (melena), grupa, mamas, pene, testículos, genitales femeninos.

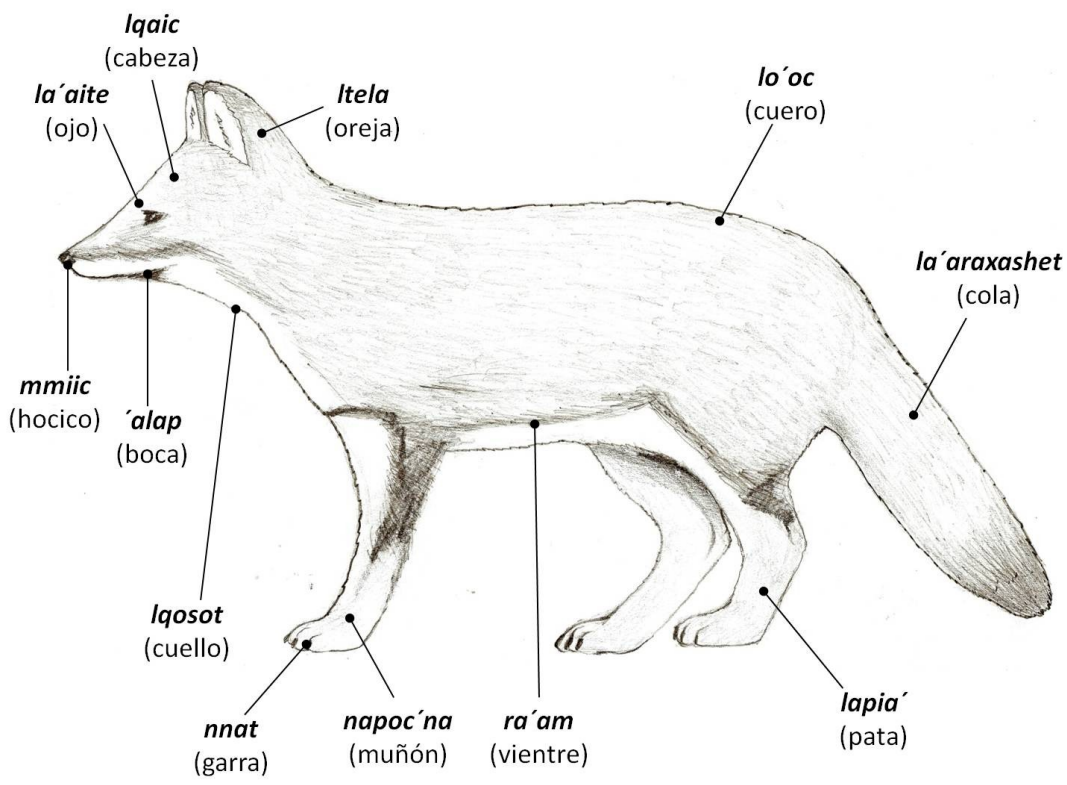




\section{FIGURA 3: Partes del cuerpo de un cánido (zorro). Indicando los nombres en castellano y los nombres en qom l'aqtaqa. (Dibujo: Ruy Gatti)}

- Patas: la pata delantera, la pata trasera, rodilla, articulación de la rodilla, corvejón, tendón, muslo, nalga. Para felinos: uñas retractiles. Para algunos carnívoros y monos: palma de la mano, dedos, uñas.

- Pie de herbívoros con pezuñas (con un dígito y un pie o con dos dígitos y dos cascos): vista lateral del pie, vista frontal de la pezuña, parte inferior del casco.

\section{1. 2. Anatomía interna}

Resulta pertinente trabajar sobre los términos relacionados con la anatomía interna cuando los hablantes retornan de una jornada de caza y se disponen a limpiar los animales para consumo. Es decir, en estas oportunidades se realizan las preguntas sobre el animal muerto pidiendo informaciones sobre las partes concretas que se van visualizando. Sin embargo, como no es frecuente presenciar estas acciones, se aporta a continuación una lista de términos que guían preguntas y una serie de dibujos que ayudan tanto al investigador para conducir la búsqueda de términos como a los hablantes en el caso de que los dibujos les parezcan familiares y no representen demasiado esfuerzo de abstracción.

Se recomienda, antes de abordar el estudio etnozoológico de algún grupo animal, realizar un estudio de la terminología académica que se maneja. En el presente trabajo se utilizó la clásica obra de Hickman Cleveland et al. (2009) como referencia para la realización de los cuestionarios sobre anatomía interna y externa, aunque cualquier Zoología General sirve para el caso.

- Corazón: sus partes. Venas: distintas categorías.

- Pulmones. Traquea (FIGURA 4). 


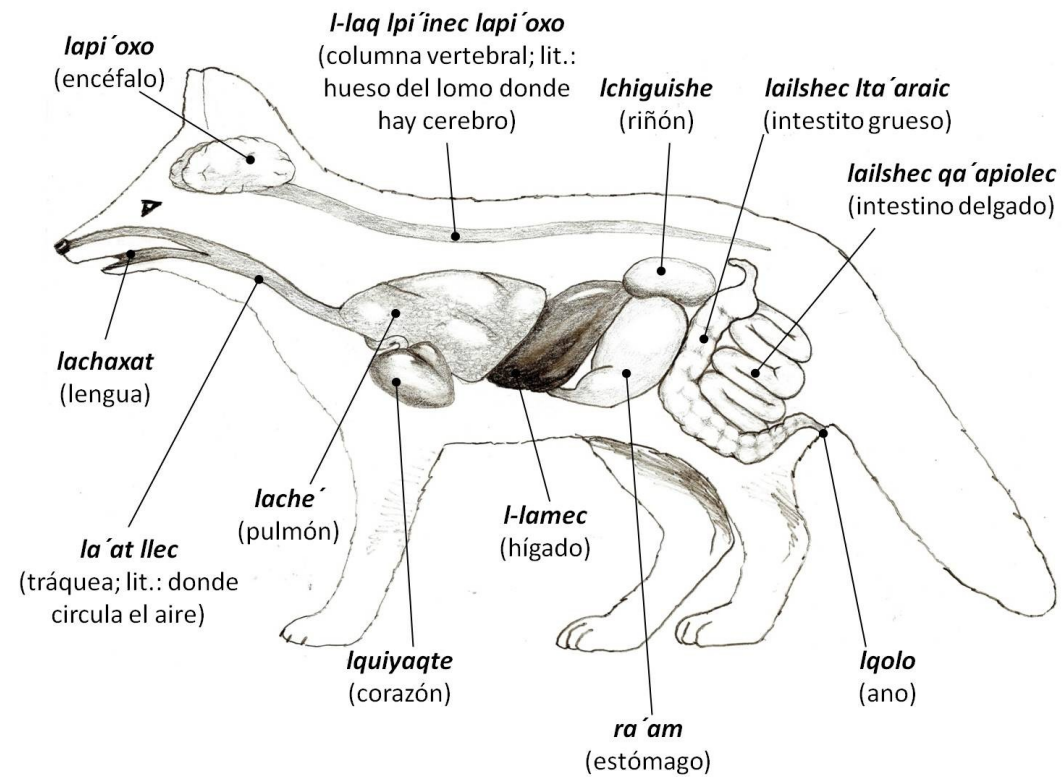

FIGURA 4: Términos que guían la búsqueda de términos inherentes a la anatomía interna de mamíferos. (Dibujo: Ruy Gatti)

- Estómago de los rumiantes: esófago, rumen (primer estómago), reticulum (segundo estómago), omasum (tercer estómago), abomasun (cuarto estómago), intestino. Estómago de los carnívoros: esófago, estómago, hígado, vesícula biliar, páncreas, intestino delgado, intestino grueso, recto, ano.

- Huesos, músculos.

\section{1. 3. Fisiología}

Si bien se pueden desarrollar los ítems relacionados con la fisiología del animal cuando se conversa sobre cada especie en particular, nos parece conveniente organizar una exploración inicial que otorgue como mínimo términos de referencia. 
- Alimentación: forma de alimentación del animal, forma de obtención del alimento (caza, pesca, recolecta), comensalidad (comen juntos, solos, alimentan a las crías), guardan alimento, partes de las presas que son ingeridas.

- Respiración: como respiran, para que sirve el aire que se respira.

- Circulación: presencia/ausencia de sangre, color de la sangre, nociones sobre la función de la sangre. Corazón: presencia/ausencia, sus funciones.

- Reproducción: intervención machos y hembras, número de crías, cuidado de las crías, construcción de nidos.

- Excreción: presencia/ausencia de excreciones, formas, lugar de depósito de las excreciones, utilización de las mismas para el reconocimiento de determinado animal.

\section{2. Atributos no físicos}

- Emociones: presencia o ausencia. Se ríe/llora. Ama/odia. Tiene miedo, ira, vergüenza.

- Capacidad de comunicación: Canta, hablar, grita, aúlla.

- Capacidad cognitiva: aprende/es inteligente/reflexiona. Es interesante realizar comparaciones con atributos que se encuentren presentes en humanos. Por ejemplo, en lengua toba se utiliza la palabra lpashec (es el entendimiento, el poder de comprender) tanto para las personas como para animales como el tigre (quiyoc) y otros que demuestran estas capacidades.

- Posee algún rasgo de "interioridad" (Descola, 2000-2001) (esto puede ser traducido como alma, espíritu, etc.) que comparte con los humanos. En lengua toba se usa la palabra lqui' $i$ para señalar aquella característica que habilita la emoción, el

\footnotetext{
${ }^{7}$ Descola define interioridad como "la gamme des propriétés ordinairement associées à l'esprit, à l'âme ou à la conscience -intentionnalité, subjectivité, réflexivité, affects, aptitude à signifier ou à rêver- mais aussi les principes immatériels supposer causer l'animation, tels le souffle ou l'énergie vitale, en même temps que des notions plus abstraites comme l'idée que je partage avec autrui une même essence, un même principe d'action ou une même origine" (2000-2001: 627) por oposición aporta el concepto de fisicalidad como a "la forme extérieure, la substance, les processus physiologiques, perceptifs et sensorimoteurs, voire le tempérament ou la façon d'agir dans le monde en tant qu'ils manifesteraient l'influence exercée sur les conduites ou les habitus par des humeurs corporelles, des régimes alimentaires, des traits anatomiques ou un mode de reproduction particuliers" (ibid.). Introducimos estos conceptos debido a que en campo encontramos datos que podrían analizarse empleando el marco teórico propuesto por el autor por lo que, comenzar a utilizar sus categorías analíticas como guía para construir nuestros cuestionarios, nos parece adecuado.
} 
conocimiento, el poder de comunicar y es compartida tanto por humanos como por nohumanos, espíritus de los muertos y dueños de los animales (Tola, 2007).

- Ciclo vital: animal joven, animal viejo, animal muerto, características propias de cada etapa. Menarca.

- Capacidad tecnológica: construye cuevas, nidos, madrigueras. Herramientas, objetos.

\section{Cuestionario 2:}

Luego de haber examinado la terminología anatómica general podemos comenzar a explorar las características de cada animal en particular. Para aportar estímulos visuales que faciliten la tarea, se pueden organizar caminatas con los informantes o salidas de pesca o caza (considerando sus riesgos y complicaciones). Sin embargo, la observación de muchas especies es ardua o las mismas se encuentran extinguidos a nivel local razón por la cual organizar álbumes de fotografías con las especies presentes en la zona puede constituir una buena herramienta de trabajo. En primera instancia debemos investigar el elenco de animales presentes en la zona utilizando publicaciones científicas y, a partir de estas listas, confeccionar las colecciones fotográficas o de dibujos.

Para el trabajo etnozoológico que se toma como ejemplo se confeccionó un álbum fotográfico que cuenta con 62 fotos de mamíferos (FIGURA 5). Las mismas fueron extraídas de guías locales como las de Parera (2002), Canevari y Fernandez Balboa (2003) y Canevari y Vaccaro (2007).

Sin embargo, la metodología de trabajo con álbum fotográfico no siempre produce los resultados deseados. Muchas veces, el despliegue de tantas fotografías genera en los hablantes ansiedades que impiden una conversación detallada sobre cada especie de animal en particular. Por otro lado, las imágenes convocan a las personas que se encuentran en el entorno y se genera un entusiasmo que, si bien aporta interesantes datos sobre las especies conocidas por los distintos miembros del grupo, imposibilita el desarrollo del cuestionario que concierne desplegar con cada una de las imágenes. Según mi propia experiencia, una manera de solucionar este inconveniente es socializar los álbumes en una instancia previa a la entrevista sobre cada especie. De esta manera, las colecciones de fotografías se vuelven más familiares y se puede desarrollar la técnica con mayor fluidez.

A continuación se listan las preguntas que se despliegan con cada una de las fotografías. 


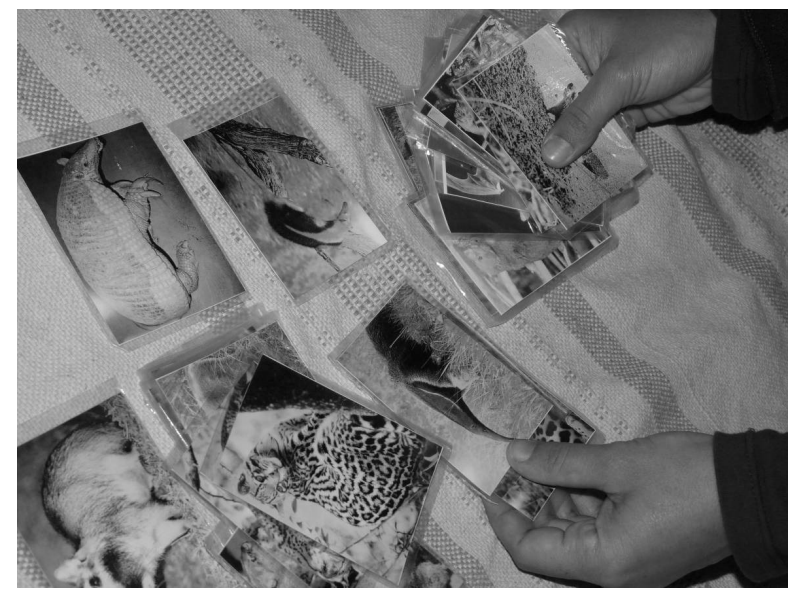

FIGURA 5: Trabajando con álbumes de fotografías de mamíferos.

\section{Los distintos animales}

\subsection{Nombres:}

- Nombre criollo.

- Nombre en qom l'aqtaqa

- Nombre/s científico/s. Puede que las especies nativas se correspondan una a una con los nombres científicos o puede que a una especies nativa le corresponda más de un nombre científico (porque se asocian varias especies bajo un mismo nombre) o viceversa.

\subsection{Datos morfológicos:}

- Color: presencia de rayas, franjas, lunares, manchas. Piel desnuda o con pelos, caparazón, placas.

- Tamaño.

- Dimorfismos sexuales: rasgos que permiten reconocerlo.

\subsection{Ubicación geográfica:}

- Donde vive. Cuanto tiempo hay que caminar para encontrarlo. Hoy se puede encontrar cerca, antes se podía encontrar cerca. Motivos de la desaparición del lugar.

\subsection{Distintas categorías de uso:}


- Comestible.

- Medicinal: Curativa/Preventiva.

- Agorera/ anunciante.

- Cultura material.

- Comercio.

- Mascota.

- Vinculo chamánico.

- Temores y prohibiciones.

- Plaga.

\subsection{Datos ecológicos:}

- Comportamiento (agresivo, manso, etc.).

- Alimentación.

- Hábitat (construye nidos, cuevas, madrigueras, vive sobre rocas, en los ríos, en los bañados).

- Reproducción (se casan, tienen hijos).

- Horas de actividad (nocturno, diurno, vespertino).

- Desplazamiento (corre, camina, nada, vuela, se arrastra).

- Sonidos que produce (ladridos, llantos, gritos, etc.) y otras huellas en el espacio (excrementos, rasguños, olores).

\subsection{Relación con el hombre:}

- Se caza: tipo de técnica empleada para su obtención, donde se obtiene, número de participantes que intervienen en la obtención, rituales que acompañan su búsqueda, presencia de dueños/dueñas de las especies al que hay que solicitarle permiso para la obtención y consumo. Momentos del año en que se lo caza.

- Se domestica: fin de la domesticación (consumo, mascotismo, fines ornamentales).

Una vez que se han explorado las características de los animales se puede intentar un boceto de la taxonomía ${ }^{8}$ que la comunidad utiliza para clasificarlos. Ésta es una tarea que implica el

\footnotetext{
${ }^{8}$ Definimos "taxonomía" la forma que tiene una sociedad de ordenar, jerarquizar y nombrar a los seres vivos. Particularmente, los distintos grupos indígenas han labrado y sostenido formas singulares de
} 
conocimiento de una amplia gama de taxones zoológicos (no solo mamíferos por ejemplo) y un extenso manejo de la lengua nativa por lo que es aconsejable realizarla casi al final de una investigación etnozoológica. No obstante, bocetos parciales nos permitirán reorganizar nuestras preguntas y nuestra búsqueda de datos en campo.

\section{EL USO DE ESTÍMULOS EN LA INVESTIGACIÓN ETNOZOOLÓGICA}

Hasta el momento los cuestionarios propuestos se basan en la presentación a los informantes de estímulos visuales (fotografías y dibujos). En etnozoología, estos instrumentos son claves cuando se pretende obtener información sobre taxas como por ejemplo, la de los mamíferos, donde desplegar ejemplares vivos o muertos sería imposible. Por otro lado, si el conjunto animal elegido está bien ilustrado dentro de los estudios biológicos clásicos, se pueden exhibir imágenes de gran parte de la diversidad zoológica para conversar con los hablantes.

Las desventajas del uso de esta herramienta se vinculan con el empleo de fotografías de mala calidad, la poca familiarización de los hablantes con las fotos, la utilización de otros sentidos para identificar especies (olor, tacto, sabor) e impedimentos físicos de los informantes como escasa visión.

Algunos de estos problemas pueden ser superados si el investigador les presenta a los informantes una diversidad de estímulos que contribuyan a confirmar la identidad zoológica de las especies de las que se quiere obtener información. Una opción interesante es por ejemplo hacer escuchar a nuestros consultantes cantos de aves cuando se trata de una investigación etnoornitológica. En otros casos se pueden utilizar colecciones de plumas o cueros de mamíferos para indagar. La desventaja de esto último es que dichos materiales generalmente no son de propiedad del investigador -sino préstamos de colecciones de museos- y deben ser trasladados con gran cuidado y responsabilidad. Esto último conduce a preocupaciones en campo que involucran devolver el material en las mismas condiciones que se lo obtuvo. Otra opción consiste en presentar a los informantes ejemplares en alcohol o formol que puede generar problemas primero a la hora de reconocer los ejemplares (decolorados y deteriorados en sus líquidos conservantes) y luego en cuanto al peligro que representa manejar estos líquidos fuera de un laboratorio?.

ordenar su entorno biológico estableciendo así etnotaxonomías o taxonomías folk que condensan visiones particulares del mundo natural.

${ }^{9}$ Sobre el tema, Arenas (2003) y Arenas y Porini (2009) desarrollaron estudios con toba y wichi en el Chaco argentino y exponen su experiencia con respecto a la utilización de colecciones de ejemplares biológicos, dibujos, fotografías y una serie de técnicas que los autores necesitaron emplear para obtener datos etnobiológicos. 
A raíz de esto, es siempre necesario testear la eficacia del recurso utilizado y evaluar sus ventajas y desventajas. Muniz de Medeiros et al. (2010) recomiendan asegurase que el estímulo utilizado sea el adecuado para la obtención de datos dentro de las circunstancias sociales, culturales y ambientales en las que se trabaja. Los mismos autores, proponen realizar ensayos para ajustar el tipo o las combinaciones de estímulos visuales más adecuados para cada situación (Muniz de Medeiros et al., ibid.: 158) y asentar estos procesos de elección en los trabajo publicados con el fin de lograr avances metodológicos en etnobiología ${ }^{10}$. Nuestra propia experiencia nos previene para no suponer que lo planeado en gabinete es una receta exacta a ser aplicada y nos entrena en el despliegue en campo de una amplia gama de técnicas que puedan responder a los intereses, capacidades, habilidades, estados emocionales, circunstancias sociales y ambientales nuestras y de los informantes.

Finalmente, una vez que se maneja la terminología zoológica y los nombres de los distintos animales en lengua nativa, se pueden desplegar cuestionarios que apunten a indagar aspectos específicos del mundo zoológico. No obstante, a medida que nos familiarizamos con la lengua y otros aspectos de la vida social y el entorno natural de nuestros consultantes, nos enfrentamos con la necesidad de profundizar nuestras preguntas. Esto implica retomar los cuestionarios anteriores y ahondar en aspectos antes no indagados. Por lo tanto, es útil tener siempre a mano todas nuestras guías de preguntas de manera que no nos sorprenda una situación en la que podríamos sacar más provecho contemplando los cuestionarios previamente elaboradas.

A continuación se presenta, a modo de ejemplo, una entrevista elaborada con el objeto de conocer aspectos relacionados con la marisca ${ }^{11}$ (caza, pesca y recolección) entre los qom $^{12}$.

\footnotetext{
${ }^{10}$ Una de las preocupaciones centrales del trabajo de Muniz de Medeiros et al. (2010) es alcanzar una terminología uniforme que describa las técnicas utilizada en los estudios etnobiológicos y proponen una estandarización para estudios etnobotánicos que se resume a continuación y que nos parece pertinente discutir para su futura adopción: "Inventarios-entrevistas: estudios en los que se acople un inventario de vegetación con un estudio etnobotánico concomitante utilizando individuos vegetales como estímulo; Walk-in-the-woods: estudios que lleven a los informantes al campo para identificar individuos independientemente de un inventario de vegetación; Artefacto-inventario: estudios que utilizan como estímulo productos derivados de vegetales como herramientas, material de construcción de casas, etc.; Checklist-entrevista: estudios en donde se pretende alcanzar conocimientos sobre una lista de plantas o animales utilizando o no estímulos visuales (ibid.: 164-165, traducción nuestra)". La propuesta debería ser discutida a la luz de investigaciones actuales adaptada para comprender también estudios etnozoológicos.
} 


\section{Cuestionario 3: Actividades de marisca ayer y hoy:}

- ¿Usted marisca? ¿Sus padres lo hacían? ¿Sus abuelos?

- ¿Por qué mariscaban antes? ¿Por qué mariscan ahora?

\subsection{Participantes:}

- ¿Quiénes iban a mariscar? ¿Los hombres a partir de qué edad? ¿Cuándo un chico podía empezar a mariscar? ¿Tenía que recibir algún conocimiento?

- ¿Qué mariscan las mujeres? ¿A partir de qué edad van las mujeres? ¿Cuándo una mujer podía empezar a mariscar? ¿Cuándo era prohibido para una mujer mariscar?

- ¿Se sale en grupo a mariscar? ¿Quién decide los integrantes de grupo? ¿En alguna oportunidad se sale solo a mariscar? ¿Se puede salir en pareja (matrimonio) a mariscar? ¿Cuándo? ¿Se puede salir en familia a mariscar? ¿Cuándo?

- ¿Quién decidía cuando había que salir a mariscar?

- ¿Quién decidía donde había que ir?

\subsection{Frecuencia:}

- ¿Cuántas veces a la semana? ¿Cuántas veces al mes?

- ¿En qué épocas del año?

- ¿Se caza de noche? ¿Cómo es una cacería nocturna?

\subsection{Productos:}

- ¿Qué traían de la marisca? ¿Algo se consumía en el monte? ¿Lo que no se consumía en el monte cómo se traía? ¿Preparaban algo en el monte?

- ¿Cuál es la diferencia con lo que se traían antes y lo que se trae ahora?

- ¿Animales: cuáles son los preferidos? ¿Cuáles son no cazados? ¿Cuáles está prohibido cazar? ¿Cuántos animales se pueden traer del monte?

\subsection{Instrumentos:}

- ¿Qué llevaban al monte para la marisca? Bolsas/ arcos y flechas/ lanzas/ trampas/ boleadoras/ fijas/ cuchillos/ mazas. Armas de fuego.

\footnotetext{
${ }^{11}$ Término utilizado por criollos e indígenas en el Chaco argentino para referir a la acción de ir a pescar, cazar o recolectar.

${ }^{12}$ Sin embargo se pueden diseñar otras que versen sobre distintos aspectos -usos medicinales de los animales, técnicas de cocción de las especies consumidas, etc.- y respondan a los intereses particulares del investigador.
} 
- Perros: ¿Se llevan? ¿Cuántos? ¿Hay algunos que sirven más para la marisca en el monte? ¿Cómo participan los perros?

- Caballo: ¿Cuándo se usa el caballo? ¿En qué tipo de marisca? ¿Quiénes usan caballo?

\subsection{Modalidades:}

- Modalidades de caza: por quema, por abatimiento.

- Modalidades de pesca.

\subsection{Percepción del monte:}

- Cuánto monte hay para cazar ahora/tiempo de los padres/ tiempo de los abuelos.

- ¿En qué parte del monte se consiguen más animales?

- Si existe poco monte o pocas presas: ¿Por qué puede ser?

\subsection{Mitos y creencias:}

- ¿Había que pedirle permiso a algún dueño del monte para cazar? ¿Qué cosas están prohibido cazar, pescar o recolectar?

- Uso de amuletos.

- Preparación del cazador antes de salir. (escarificaciones, rezos, cantos). Vestimenta específica del cazador. Adornos. Perfumes.

\subsection{Uso de las presas:}

- ¿Cómo se preparan los animales mariscados? ¿Quiénes los preparan? ¿Qué partes se consumen y cuales no? ¿Cuáles animales son tufientos? ¿Cuáles está prohibido consumir?

\section{LA NECESIDAD DE COLECTAS BIOLÓGICAS}

Si bien en etnobiología se utilizan cuestionarios cuando se desea conocer la manera en que una sociedad se relaciona con su entorno natural, la particularidad de la disciplina radica en anexar a estas indagaciones datos inherentes a la identidad taxonómica "occidental" de las especies sobre las que se aportan datos. Al ser compartida académicamente, esta identidad permite realizar comparaciones y establecer un corpus coherente de datos.

Por ejemplo, si examinamos el conocimiento nativo sobre especies de abejas nativas, a la información obtenida en campo le anexamos el nombre científico del animal al cuál el hablante se refiere. A continuación aportamos una muestra esclarecedora:

Isabel: "Cuando una mujer se enferma (menstrúa) no puede tocar agua ni acercarse o tampoco estar en contacto con humedad. Otra cosa muy peligrosa es nsherec [Meliponini: Geotrigona argentina]. No hay que acercarse cuanto está enferma porque va a aparecer el arco iris y se va a 
hundir todo. Pero los criollos a veces no saben, ellos no saben y por eso pasan cosas" ( $\mathrm{B}^{\circ} \mathrm{La}$ Paz, Formosa. Abril/2010).

En este ejemplo, Isabel narra uno de los tabúes vinculados con la menstruación femenina ${ }^{13}$. Gracias a la colecta de material biológico en la zona y a su identificación científica, pudimos asociar el nombre en lengua qom (nsherec) a una especie de abeja sin aguijón (Meliponini: Geotrigona argentina), nativa del continente americano, que realiza nidos bajo tierra de los cuales los indígenas chaqueños aprovechan miel. En el futuro, otros investigadores podrán recolectar más información referida a nsherec o a G. argentina y compartir sus datos teniendo la certeza de estar asociándolos al mismo insecto.

Sin embargo, colectar ejemplares biológicos no es una tarea sencilla y existen reglas que deben seguirse para evitar perder el material recolectado por falta de datos o mala conservación. Por esto, antes de realizar una investigación etnozoológica, es necesario adquirir un entrenamiento en estos temas o bien, trabajar conjuntamente con biólogos, quienes realizarán buenas colectar y podrán identificar el nombre científico de los ejemplares colectados o reconocer cuando se presenta la necesidad de recurrir a especialistas.

\section{CONSIDERACIONES FINALES}

Actualmente múltiples estudios etnozoológicos se están desarrollando en comunidades indígenas donde generalmente las personas manejan una lengua diferente a la del investigador $\mathrm{y}$, por lo tanto, resulta clave desarrollar técnicas que nos permitan dialogar utilizando la terminología adecuada. Aprender la lengua se traduce en un gesto que nos va a permitir comprender realmente los mundos del otro, su forma de concebir la fauna, de clasificarla, de sentirla y la manera en que ésta participa en diversos aspectos de la vida social.

Nuevos escenarios de trabajo se están abriendo en contextos rurales y urbanos donde los hablantes bilingües poseen distintos grados de competencia en el español. En estos últimos espacios, no debemos asumir a priori que los hablantes, comparten nuestra misma terminología para referirse al entorno natural. En dichos contextos, una adecuada exploración de los atributos, de las características zoológicas y de la taxonomía empleada por la comunidad nos puede sorprender y rebatir nuestros presupuestos iniciales de encontrar semejanzas por el simple hecho del manejo de una lengua afín.

Por otro lado, no debemos abusar de herramientas como los cuestionarios y pensar que lo que uno tiene en mente antes de salir al campo puede no coincidir con la realidad etnográfica.

\footnotetext{
${ }^{13}$ Sobre los tabúes relacionados a la menstruación femenina, cf. Karsten (1923) para los toba de Bolivia, Métraux (1946) para los toba y pilagá y Cordeu (1969-70) para los toba.
} 
Debemos permitirnos echar por tierra la metodología pergeñada en gabinete si con los hablantes solo encontramos incomodidad como toda respuesta y probar con otro cuestionarios o bien entender que, si bien resulta el más legítimo de los métodos, muchas veces no coopera en el establecimiento de un vínculo de mutuo conocimiento entre el investigador y el informante.

No debemos desestimar otros métodos etnográficos que incluyen desde la clásica observación participante a la elaboración de esquemas y mapas participativos, las caminatas etnobiológicas, la participación conjunta en proyectos audiovisuales. Sin bien resulta obvio que debemos consensuar los objetivos de nuestra investigación con la comunidad y programar instancias de devolución de los resultados, muchas veces se aplican cuestionarios salteando esta etapa lo que puede generar desconfianza, antipatía y sobre todo podemos correr el riesgo de llegar a conclusiones erradas si nos empecinaos en emplear cuestionarios pre elaborados sin sostener una permanente vigilancia de los desaciertos en los que nos hacen caer los abusos de los mismos.

El quid es construir el método con los hablantes según sus propias necesidades y preocupaciones con la certeza de encontrar así, el camino hacia un conocimiento genuino que nos permita edificar verdaderamente esta ciencia del diálogo de saberes.

\section{AGRADECIMIENTOS}

A la antropóloga Florencia Tola y a la lingüista Cristina Messineo por la lectura crítica del manuscrito. A Ruy Gatti por la elaboración de los dibujos que figuran en este texto.

\section{BIBLIOGRAFÍA}

ALBUQUERQUE, U. (1999): "La importancia de los estudios etnobiológicos para el establecimiento de estrategias de manejo y conservación en las florestas tropicales" -Biotemas 12 (1)- Santa Catarina, Brasil (págs 31-47)

ARENAS, P. (2003): Etnografía y alimentación entre los toba-ñachilamoleek y wichi-lhuku'tas del Chaco Central (Argentina)- Edición del autor -Buenos Aires.

ARENAS, P. y PORINI, G. (2009): Las aves en la vida de los tobas del oeste de la provincia de Formosa (Argentina)- Editorial Tiempo de Historia -Asunción.

BROWN, R. (1868): “On the vegetable products, used by the Northwest American Indians as food and medicine, in the arts, and in superstitions rites"- Transactions of the Botanical Society of Edinburgh, 9- Edinburgh- (págs 378-396).

CANEVARI, M. y FERNANDEZ BALBOA, C. (2003): 100 Mamiferos Argentinos- Editorial Albatros- Buenos Aires. 
CANEVARI, M. y VACCARO, O. (2007): Guía de mamíferos del sur de América del SurL.O.L.A- Buenos Aires.

CASTETTER, E. F. (1935): "Ethnobiological studies in the American Southwest, I. Uncultivated native plants used as sources of food"- University of New Mexico Bulletin, 4México (págs 1-44).

CORDEU, E. (1969-70): “Aproximación al horizonte mítico de los Toba"- Runa, 12 (1-2)Buenos Aires (págs 67-176).

COSTA NETO, E. M., VARGAS CLAVIJO, M. y SANTOS FITA, D. (2009): "Introducción" en Manual de Etnozoología- Eraldo Medeiro Costa Neto, Dídac Santos Fitas y Mauricio Vargas Clavijo (EDS)- Tundra ediciones- Valencia.

CLÉMENT, D. (1998): The historical fundations of etnobiology (1860-1899)- Journal of Ethnobiology, 18 (2)- Texas (págs 161-187).

DESCOLA, P. (2000-2001): Anthropologie de la Nature. Résumé des cours et travauxAnnuaire du Collège de France- París (págs 627-644).

DIEGUES, C. (2000): "Etnoconservação da Natureza: enfoques alternativos" en Etnoconservação: novos rumos para a proteção da natureza nos trópicos- Antonio Carlos Diegues (ED).- Editora Hucitec- São Paulo.

GUBER, R. (2009): El salvaje metropolitano. Reconstrucción del conocimiento social en el trabajo de campo- Paidós- Buenos Aires.

HENDERSON , J. y HARRINGTON, J. P. (1914): Ethnozoology of the Tewa IndiansBureau of American Ethnology Bulletin, 56- Noth América (págs 1-76).

HICKMAN CLEVELAND P., EISENHOUR DAVID J., KEEN SUSAN L., L'ANSON HELEN, LARSON ALLAN y ROBERTS LARRY S. (2009): Principios integrales de Zoología (14º edición)- MCGRAW-HILL- España.

HUNN, E. (2007): Ethnobiology in four phases- Journal of Ethnobiology, 27 (1)- Texas (págs 339-367).

KARSTEN, R. (1932): Indian Tribes of the Argentine and Bolivian Chaco- Ethnological Studies. Societas Scientiarum Fennica, 4 (1)- Helsingfors (págs 10-236).

MASON, O. T. (1989): Aboriginal American zootechny- American Anthropologist, 1 (1)North America (págs 45-81).

MÉTRAUX, A. (1946): Myth of the Toba and Pilagá Indians of the Gran Chaco- American Folklore Society- Philadelphia.

MUNIZ DE MEDEIROS, P., SANTOS DE ALMEIDA, A. L., FARIAS PAIVA DE LUCENA, R., BEZERRA SOUTO, F. J. y ALBUQUERQUE, U. (2010): "Uso de estímulos visuais na pesquisa etnobiológica" en Métodos e Técnicas na Pesquisa Etnobiológica e 
Etnoecológica- Ulyseses Paulino de Albuquerque, Reinaldo Farias Paiva de Lucena y Luiz Vital Fernandez Cruz de Cunha (EDS)- NUPPEA- Recife.

PALMER, E. (1878): Plants used by the Indians of the United States- The American Naturalist, 12 (9)- Noth America (págs 593-606).

PARERA, A. (2002): Los mamíferos de la Argentina y la región austral de Sudamérica- El ateneo- Buenos Aires.

POSEY, D. A. (1987): "Etnobiología: teoría e prática" en Suma etnológica, 1. EtnobiologíaBelém Ribeiro (ED)- Vozes/Finep- Petrópolis.

ROBBINS, W. W, HARRINGTON, J. P. y FREIRE-MARRECO, B. (1916): Ethnobotany of the Tewa Indians- Bureau of American Ethnology Bulletin, 55- Noth America (págs 1-118).

SANTOS FITA, D., COSTA NETO, E. M. y CANO-CONTRERAS, E. J. (2009): "El quehacer de la etnozoología" en Manual de Etnozoología- Eraldo Medeiro Costa Neto, Dídac Santos Fitas y Mauricio Vargas Clavijo (EDS)- Tundra ediciones- Valencia.

SANTOS RODRÍGUEZ, A. (2009): "Metodología de la investigación etnozoológica" en Manual de Etnozoología- Eraldo Medeiro Costa Neto, Dídac Santos Fitas y Mauricio Vargas Clavijo (EDS)- Tundra ediciones- Valencia.

SUSNIK, B. (1972): Dimensiones migratorias y pautas culturales de los pueblos del Gran Chaco y su periferia- Instituto de Historia, Facultad de Humanidades, Universidad Nacional del Nordeste- Resistencia.

THOMAS, J. (1992) : "Ethnozoology" en Studying and Describing Unwritten Languages- Luc Bouquiaux y Jacqueline M. C. Thomas (EDS)- International Academic Bookstore, SIL International- Dallas.

TOLA, F. (2007): "Eu não estoy só(mente) em meu corpo" A pessoa e o corpo entre os Toba (Qom) do Chaco argentino- Mana 13 (2)- Brasil (págs 499-519).

TOLEDO, V.M.

(1990): La perspectiva etnoecológica. Ciencias, Especial 4- México (págs 22-29).

(1991): El juego de la supervivencia. Un manual para la investigación etnoecológica en Latinoamérica- Consorcio Latinoamericano sobre agroecología y Desarrollo (CLADES).

TOWSEND, W. (1996): Taller participativo sobre manejo de fauna silvestre en Lomerio. Proyecto de Manejo Forestal Sostenible- BOLFOR, USAID, gobierno de Bolivia, International Chemonics, International Conservation, Tropical Research and Development y Wildlife Conservation Society- Santa Cruz, Bolivia.

ULLOA, A., RUBIO, H. y CAMPOS, C. (1996): "Trua Wandra”. Estrategias para el manejo de la fauna con comunidades Embera en el parque Nacional Natural de Utría, Chocó, Colombia- Fundación Natura- Bogotá. 
VENOT, C. (1992): "Anatomy and physiology, Animals and plants" en Studying and Describing Unwritten Languages- Luc Bouquiaux y Jacqueline M. C. Thomas (EDS)International Academic Bookstore, SIL International- Dallas. 